Pacific Journal of Mathematic 


\title{
A CYCLIC INEQUALITY AND A RELATED EIGENVALUE PROBLEM
}

\author{
J. L. Searcy and B. A. Troesch
}

A cyclic sum $S(\underline{x})=\Sigma x_{i} /\left(x_{i+1}+x_{i+2}\right)$ is formed with the $N$ components of a vector $\underline{x}$, where $x_{N+1}=x_{1}, x_{N+2}=x_{2}$, and where all denominators are positive and all numerators nonnegative. It is known that the inequality $S(\underline{x}) \geqq N / 2$ does not hold for even $N \geqq 14$; this result is derived in a uniform manner by considering a related algebraic eigenvalue problem. Numerical evidence is presented for the conjecture that this cyclic inequality is true for even $N \leqq 12$ and odd $N \leqq 23$.

The corresponding cyclic inequality, namely the question for what value of $N$

$$
S(\underline{x}) \geqq N / 2
$$

holds, has been investigated by many mathematicians (cf. Mitrinović [7] and the references given there). In $\S 1$ we prove in a unified manner that the inequality does not hold for even $N \geqq 14$. The method is based on the idea used first by Lighthill for $N=20$ [4] and then by several other authors. The argument indicates why the case $N=12$ remains still unresolved. Some properties of this type of solution are described in $\S 2$. Section 3 deals with numerical results that strongly suggest that the inequality is valid for $N=12$ and, if $N$ is odd, for $N=23$. These numerical results definitely represent stationary values of the cyclic sum, and we are inclined to believe that they are indeed global minima. A connection between the inequality above and a related inequality with indices reversed is considered in the last section. In the Appendix some examples are listed for $N=14,25$ and 27.

1. The linear cyclic inequality. By considering the cyclic sum $S(\underline{x})$ it is obvious that for any $N$ there exists a vector for which

$$
S(\underline{x})=N / 2
$$

holds, namely $x_{i}=1$ for $i=1,2, \cdots, N$. If $N$ is even, there exists also a wider class of "nominal" vectors,

$$
x_{i}^{0}=\left\{\begin{array}{ll}
(1+\alpha) / 2 & \text { for } i \text { odd } \\
(1-\alpha) / 2 & \text { for } i \text { even }
\end{array} 0 \leqq \alpha \leqq 1,\right.
$$


for which $S\left(\underline{x}^{0}\right)=N / 2$. Vectors of this type seem to form the basis in the reported solutions for even $N$ where the inequality does not hold, in particular, in Zulauf's solution [7, p. 133] for the important case $N=14$.

If $N$ is odd, the situation is much more difficult to understand. Indeed, while only $N=12$ is unresolved for even $N$, for odd $N$ the answer is still unknown for $N=11,13, \cdots, 23$. A simple nominal vector of the form (1.1) exists for odd $N$ only if $\alpha=0$.

We now show in a uniform manner that the cyclic inequality is violated for even $N \geqq 14$. (In the remainder of this section, $N$ is understood to be even.) ' We proceed by writing the vector $\underline{x}$ as $\underline{x}=\underline{x}^{0}+\underline{e}$ and expanding the cyclic sum $S(\underline{x})$ in terms of the components of the vector $\underline{e}$. If $S$ can be made smaller than $N / 2$ for small $e$, the inequality is clearly violated.

By including quadratic terms in the expansion-the contribution of the linear terms vanishes-we obtain

$$
S^{*}=N / 2+\sum e_{k}^{2}-e_{k} e_{k+2}+(-1)^{k} \alpha e_{k} e_{k+1}=N / 2+e^{T} A e / 2
$$

where again $e_{N+1}=e_{1}, e_{N+2}=e_{2}$ and where $A$ is the symmetric matrix

$$
A=\left(\begin{array}{rrrrrrrrrr}
2 & -\alpha & -1 & & & & & -1 & \alpha \\
-\alpha & 2 & \alpha & -1 & & & & & -1 \\
-1 & \alpha & 2 & -\alpha & -1 & & & & \\
& & & - & - & & & & & \\
& & & & -1 & -\alpha & 2 & \alpha & -1 \\
-1 & & & & -1 & \alpha & 2 & -\alpha \\
\alpha & -1 & & & & & -1 & -\alpha & 2
\end{array}\right)
$$

In order to minimize $S^{*}$ we must minimize $\underline{e}^{T} A \underline{e}$ with $\underline{e}^{T} \underline{e}$ kept constant. The corresponding eigenvalue problem $(\bar{A}-\lambda I) \underline{e}=\underline{0}$ has the known solution, which can be easily verified,

$$
e_{k}=\left\{\begin{aligned}
a \sin t_{k} & \text { for } k \text { odd } \\
-a \cos t_{k} & \text { for } k \text { even }
\end{aligned}\right.
$$

where $t_{k}=t_{0}+(k-1) h$; the amplitude $a>0$ and the phase $t_{0}$ are arbitrary, and

$$
h=2 \pi j / N, \quad j=1,2, \cdots, N .
$$

The $N$ corresponding eigenvalues are

$$
\lambda=2 \sin h(2 \sin h-\alpha) ;
$$


they are, with the exception of at most two of them, all double eigenvalues. We may choose $t_{0}=0$ so that the $\underline{e}$-vector becomes

$$
\underline{e}=a(0,-\cos h, \sin 2 h,-\cos 3 h, \cdots, \sin (N-2) h,-\cos (N-1) h) .
$$

Now, at the stationary values of $S^{*}$ we have

$$
S^{*}=N / 2+\lambda \underline{e}^{T} \underline{e} / 2 \text {. }
$$

Hence, $S^{*}$ is smaller than $N / 2$ if there exists at least one negative eigenvalue $\lambda$. This means that we must require that $0<2 \sin h<$ $\alpha<1$, i.e., $0<\sin (2 \pi j / N)<1 / 2,2 \pi j / N<\pi / 6$, or finally $N>12 j$. The case where $5 \pi / 6<2 \pi j / N<\pi$ can be excluded since it leads to the indentical result for $\underline{x}$ and $S^{*}$. For $N>12$, the condition $N>$ $12 j$ can indeed always be satisfied. We conclude that vectors of this kind with $S^{*}<N / 2$, and therefore also for the full cyclic inequality with $S<N / 2$, are always possible for $N \geqq 14$, but not possible for $N \leqq 12$ (cf. also [10]). This concludes the main argument.

However, these considerations do not resolve the open case $N=12$. The inequality holds in the neighborhood of a nominal vector $\underline{x}_{0}$. Consequently, if a vector $\underline{x}$ exists that violates the inequality, then it cannot be obtained by a perturbation of a nominal vector $\underline{x}^{0}$.

2. The minimum of the linear cyclic sum. It seems worthwhile to elaborate on the vectors formed with (1.2) and add a few remarks.

First, we note that $\lambda=4 \sin ^{2} h \geqq 0$ for $\alpha=0$. This means that for odd $N$, where the only simple nominal vector $x^{0}$ is furnished by $\alpha=0$, the eigenvalues are all nonnegative, so that the argument given above cannot be applied to odd $N$. Furthermore, higher order terms in the $\underline{e}$-expansion do not alter this conclusion.

For $N \geqq 14$ there exists a negative eigenvalue, namely exactly one for $14 \leqq N \leqq 24$. If $24<N \leqq 36$ both $j=1$ and $j=2$ furnish negative eigenvalues, and similarly for larger $N$ values, where for each increase of $N$ by 12 a "higher harmonic" is added. The Figure 1 shows the eigenvectors for $N=26, j=1$ and $j=2$. The values of the full (i.e., not linearized) cyclic sum for these vectors are $S=13-0.01913$ and $S=13-0.0000787$.

Since all $x_{k}$ are required to be nonnegative, the amplitude $a$ must be chosen sufficiently small, namely

$$
a \leqq(1-\alpha) / 2 \text {. }
$$

In some cases, $a$ can be chosen slightly larger, e.g., for $N=14$ 

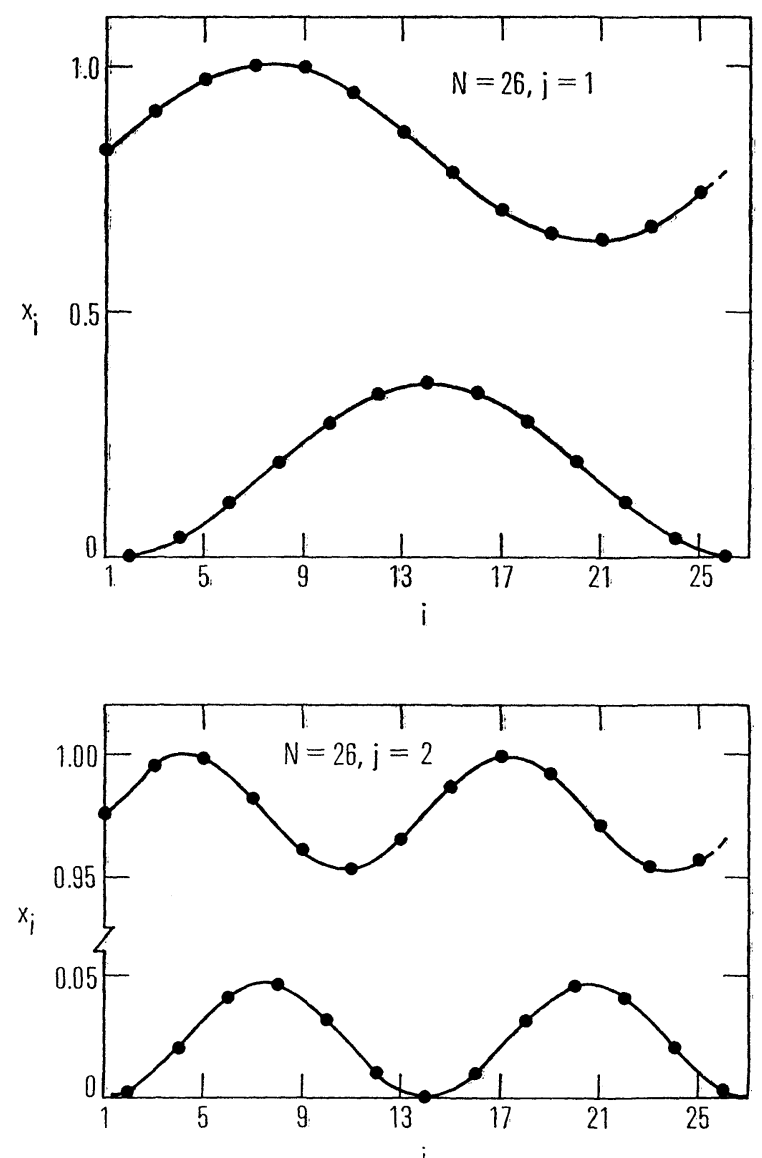

Figure 1. Eigenvectors for $N=26, j=1,2$.

and $j=1$,

$$
a \leqq(1-\alpha) / 2 \cos h,
$$

since the trigonometric functions in (1.2) are evaluated only at discrete points.

The sum $S^{*}$ is computable in closed form and gives, for the cases of interest,

$$
S^{*}=N\left(2+\lambda a^{2}\right) / 4
$$

or, using the (nearly) largest admissible $a$,

$$
S^{*}(\alpha)=N\left(2-\frac{1}{2}(1-\alpha)^{2} \sin h(\alpha-2 \sin h)\right) / 4 .
$$

For $\alpha=1$ and $\alpha=2 \sin h$, we obtain $S^{*}=N / 2$, and $S^{*}$ attains its minimum value (for either (1.3) or (1.4)) at

$$
\alpha_{0}=(1+4 \sin h) / 3 \text {, }
$$


namely

$$
S^{*}=N\left(1-\frac{1}{27} \sin h(1-2 \sin h)^{3}\right) / 2
$$

The linearized sum $S^{*}$ has of course a different minimum than the full cyclic sum. As an example, we choose $N=14, j=1$. From (1.5) we obtain for $a=(1-\alpha) / 2$

$$
S^{*}=7-0.000260,
$$

and it can be shown that for $a=(1-\alpha) / 2 \cos h(1.5)$ gives

$$
S^{*}=7-0.000320
$$

while the full cyclic sum for this vector is

$$
S=7-0.000323
$$

On the other hand, a numerical minimization of the full cyclic sum furnishes

$$
S=7-0.000347
$$

It is not difficult to include the cubic terms in the e-expansion. It turns out that in order to obtain this sum, let us call it $S^{* *}$, one only needs to increase the amplitude $a$. However, the amplitude is in general restricted to $a \leqq(1-\alpha) / 2$. Hence, it seems reasonable to increase $a$, except that those $x_{k}$ which would become negative are replaced by zero. A computation then leads to the result

$$
S^{* *}=7-0.000331 \text {. }
$$

One might expect that for large $N$ where more than one negative eigenvalue occurs, the eigenvalue for $j=1$ would give the smallest sum $S^{*}$. However, (1.5) shows that for $N \geqq 74$ this is not the case.

3. The cases $N=12$ and $N=23$. By considering the numerical minimization for $N \geqq 14$ (cf. Figure 2 and Table 1) we are led to the conjecture that for the still open case $N=12$ the inequality is indeed satisfied. But it should be kept in mind that these numerical results have not been shown to be global minima.

Similarly, for $N$ odd and larger than 23, the numerical results indicate that the inequality is valid for $N=23$. Here the solution for $N=23$ which is similar in structure to the solutions for $N \geqq 25$ is also listed, although in this case the vector $x_{k}=1$, for all $k$, furnishes the lower value $N / 2$. The same conclusion has been reached by Malcolm [6] who solved the problem for $N=25$ by 

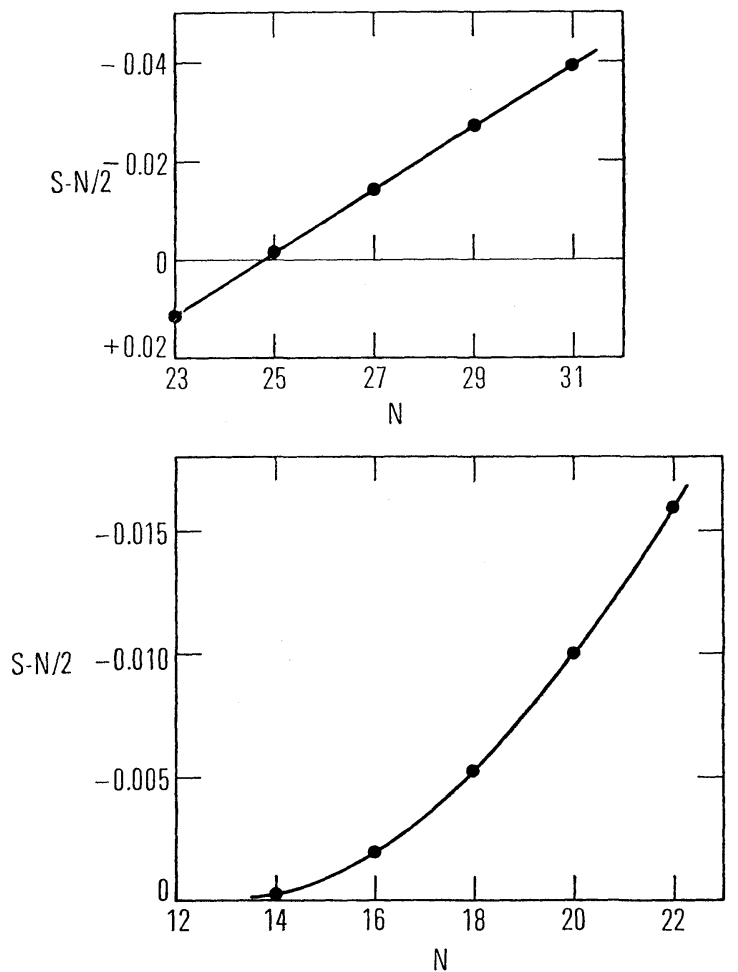

FIGURE 2. Extrapolation of the minimum cyclic sum to $N=12$ and $N=23$.

TABLE 1

Extrapolation of the minimum of the cyclic sum $S$ to $N=12$ and $N=23$.

\begin{tabular}{lccc}
\hline$N$ & $S-N / 2$ & $N$ & $S-N / 2$ \\
14 & -.000347303 & 23 & +.011689438 \\
16 & -.002004523 & 25 & -.001514765 \\
18 & -.005287982 & 27 & -.014469580 \\
20 & -.010062465 & 29 & -.027056111 \\
22 & -.015979281 & 31 & -.039127154 \\
\hline
\end{tabular}

convincing numerical minimization and by Daykin [1] who also lists a solution in integer values for the $x_{i}$.

Additional numerical results are discussed in the Appendix.

4. The cyclic inequality with indices reversed. The solutions listed above exhibit an interesting general property. We define a vector $\underline{b}$ by setting

$$
b_{i}=x_{i} /\left(x_{i+1}+x_{i+2}\right)^{2}
$$

and introduce also

$$
r_{i}=b_{i} /\left(b_{i-1}+b_{i-2}\right)
$$


as a counterpart to

$$
s_{i}=x_{i} /\left(x_{i+1}+x_{i+2}\right) \text {. }
$$

At the stationary values of $S(\underline{x})$ for admissible vectors $\underline{x}$, either $x_{i}=0$ or $\partial S / \partial x_{i}=0$. This leads readily to the relations that either

$$
\left(x_{i+1}+x_{i+2}\right)\left(b_{i-1}+b_{i-2}\right)=1 \text { or } x_{i}=b_{i}=0,
$$

and hence,

$$
\begin{gathered}
x_{i}=b_{i} /\left(b_{i-1}+b_{i-2}\right)^{2} \\
r_{i}=b_{i}\left(x_{i+1}+x_{i+2}\right)=x_{i}\left(b_{i-1}+b_{i-2}\right)=s_{i}
\end{gathered}
$$

and

$$
x_{i} b_{i}=s_{i}^{2}=r_{i}^{2}
$$

for all $i$.

Clearly then, for any stationary solution $\underline{x}^{(1)}$ another stationary solution $\underline{x}^{(2)}$ can be formed, namely the vector $\underline{b}$ read in reverse order. Both solutions lead to the same stationary sum $S=\Sigma s_{i}=$ $\Sigma r_{i}$. Therefore, if the minimum of $S$ is unique, the two vectors must be equivalent, i.e., $\underline{x}^{(2)}$ must be constant multiple of $\underline{x}^{(1)}$. The computation of many minima for both even and odd $N$ showed that in all cases indeed, $\underline{x}^{(2)}=c \underline{x}^{(1)}$. As an example we list in the Appendix, Table 4 , the results for $N=25$ where $\underline{x}^{(1)}$ has been normalized so that $c=1$, i.e., $b_{i}=x_{N+2-i}$ and $s_{i}=s_{N+2-i}$.

This means that for all computed minima (including the result in [6]) the vector $\underline{s}$ exhibits a symmetry, and it might be of interest to prove this property, if indeed it holds in general.

Since the difficult cases where the cyclic inequality holds, namely $N=8$ [3] and $N=10$ [8], have been proved by discussing all relevant possibilities in turn, the symmetry in $\underline{s}$ might just restrict the number of cases sufficiently to make $N=12$ amenable to a proof.

Appendix. Miscellaneous numerical results. In this appendix we present examples and computational results for the cyclic inequality.

The approach described in $\S 1$ enables us to obtain vectors $\underline{x}$ for which $S(\underline{x})<N / 2$ without requiring an extensive search on a computer. In Table 2 we present the results for the vector $\underline{x}_{z}$ [7, p. 133], $\underline{x}_{H}$ [5], and the vector $\underline{x}$ suggested by (1.2). For the expansion for small $e$, one obtains $S(\underline{x})=N / 2-q e^{2}+0\left(e^{3}\right)$. The minimum of the cyclic sum for these vectors is also listed; the comparison 
TABLE 2

Vectors $x$ with $S(\underline{x})<N / 2$ for small $e$. $\quad N=14$.

$x_{Z}=(1+7 e, 7 e, 1+4 e, 6 e, 1+e, 5 e, 1, \quad 2 e, 1+e, 0,1+4 e, e, 1+6 e, 4 e)$

$\underline{x}_{H}=(1+10 e, 7 e, 1+8 e, 10 e, 1+3 e, 10 e, 1-2 e, 5 e, 1-2 e, 0,1, \quad 0,1+8 e, 3 e)$

$x=(1+11 e, 8 e, 1+8 e, 10 e, 1+3 e, 8 e, 1, \quad 3 e, 1+2 e, 0,1+6 e, 0,1+10 e, 4 e)$

\begin{tabular}{lrll}
\hline vector & $q$ & $\begin{array}{l}\text { minimum } \\
\text { of } S-N / 2\end{array}$ & at $e=$ \\
$\underline{x}_{Z}$ & 2 & -0.0000215 & 0.0059 \\
$\underline{x}_{I I}$ & 3 & -0.0000028 & 0.0017 \\
$\underline{x}$ & 11 & -0.0002661 & 0.0093 \\
\hline
\end{tabular}

between $\underline{x}_{z}$ and $\underline{x}_{I I}$ shows that a larger $q$ need not lead to a smaller minimum.

The expansion in small $e$ is not available for odd $N$. Convincing examples for $S(x)<N / 2$ are then furnished by vectors with nonnegative integers as components. Table 3 lists examples for $N=14,25,27$. Clearly, there is a limit on how small the largest integer component can be chosen. We believe that the examples are quite close to optimal in this respect. The vector $x_{D}$ for $N=$

TABLE 3

Vectors $\underline{x}$ with integer components and $S(\underline{x})<N / 2$.

\begin{tabular}{|c|c|c|c|}
\hline \multicolumn{4}{|c|}{$\begin{array}{l}\underline{x}_{1}=(0,42,2,42,4,41,5,39,4,38,2,38,0,40) \\
\underline{x}_{2}=(0,44,2,44,4,43,5,41,4,40,2,40,0,42) \\
\underline{x}_{D}=(3,6,2,6,1,6,0,7,0,8,0,9,0,10,0,11,1,12,3,11,5,9,6,7,6,5,6) \\
\underline{x}_{3}=(3,5,2,5,1,5,0,6,0,7,0,8,0,9,0,10,1,11,3,10,5,8,5,6,5,4,5)\end{array}$} \\
\hline vector & $N$ & Largest $x_{i}$ & $S-N / 2$ \\
\hline$\underline{x}_{1}$ & 14 & 42 & $-151 / 28938140=-0.00000522$ \\
\hline$\underline{x}_{2}$ & 14 & 44 & $-217 / 4280760=-0.00005069$ \\
\hline Table $4, x_{\text {int }}$ & 25 & 35 & $=-0.00013752$ \\
\hline$\underline{x}_{\mathrm{int}} *$ & 25 & 35 & $-691 / 80013480=-0.00000863$ \\
\hline$\underline{x}_{D}$ & 27 & 12 & $-53 / 55440=-0.00095599$ \\
\hline$\underline{x}_{3}$ & 27 & 11 & $3465=-0.00230880$ \\
\hline$\underline{x}_{3} *$ & 27 & 11 & $126=-0.00079365$ \\
\hline
\end{tabular}

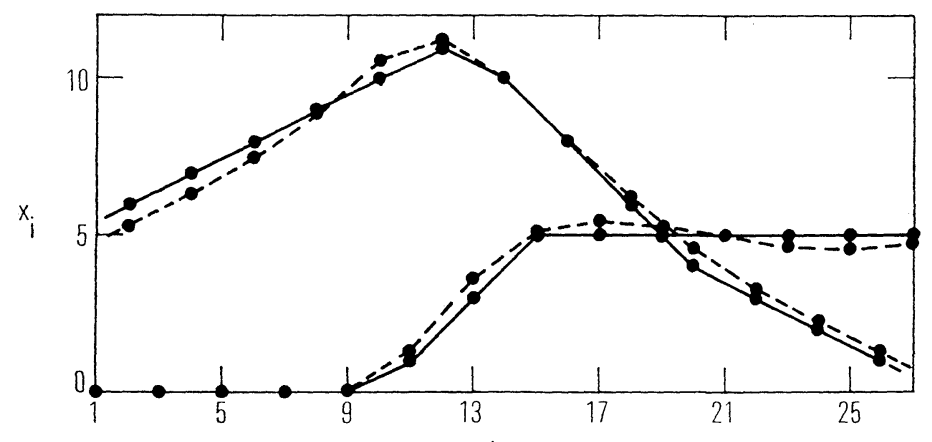

FIGURE 3. The numerical minimization of $S .-. .$, and an example with integer components $x_{i}-$ for $N=27$. 
TABLE 4

The numerical minimization of $S(x)$ for $N=25$ and a case $\underline{x}_{\mathrm{in}}$ with integer components.

\begin{tabular}{|c|c|c|}
\hline & $s$ & $x_{\mathrm{int}}$ \\
\hline$x_{1}=b_{1}=.8448196$ & .8448196 & 25 \\
\hline$x_{2}=b_{25}=.0$ & .0 & 0 \\
\hline$x_{3}=b_{24}=1.0$ & .8448196 & 29 \\
\hline$x_{4}=b_{23}=.0$ & .0 & 0 \\
\hline$x_{5}=b_{22}=1.1836847$ & .8448196 & 34 \\
\hline$x_{\ell}=b_{21}=.1924932$ & .1160666 & 5 \\
\hline$x_{7}=b_{20}=1.2086162$ & .8133369 & 35 \\
\hline$x_{8}=b_{19}=.4498554$ & .2777040 & 13 \\
\hline$x_{\vartheta}=b_{18}=1.0361416$ & .7447432 & 30 \\
\hline$x_{10}=b_{17}=.5837685$ & .4125654 & 17 \\
\hline$x_{11}=b_{16}=.8075051$ & .6676996 & 24 \\
\hline$x_{12}=b_{15}=.6074671$ & .5125019 & 18 \\
\hline$x_{13}=b_{14}=.6019168$ & .5925761 & 18 \\
\hline$x_{14}=b_{13}=.5833803$ & .5925761 & 17 \\
\hline$x_{15}=b_{12}=.4323827$ & .5125019 & 13 \\
\hline$x_{18}=b_{11}=.5520990$ & .6676996 & 16 \\
\hline$x_{17}=b_{10}=.2915714$ & .4125654 & 9 \\
\hline$x_{18}=b_{9}=.5352959$ & .7447432 & 16 \\
\hline$x_{18}=b_{8}=.1714317$ & .2777040 & 5 \\
\hline$x_{20}=b_{7}=.5473341$ & .8133369 & 16 \\
\hline$x_{21}=b_{6}=.0699841$ & .1160666 & 2 \\
\hline$x_{22}=b_{5}=.6029648$ & .8448196 & 18 \\
\hline$x_{23}=b_{4}=.0$ & .0 & 0 \\
\hline$x_{24}=b_{3}=.7137202$ & .8448196 & 21 \\
\hline$x_{25}=b_{2}=.0$ & .0 & 0 \\
\hline
\end{tabular}

$S(x)=12.498485$

27 is published in [2], and the vector $\underline{x}_{\text {int }}$ is a slight modification of the vector given in [9] (the authors were unaware of the results in [1] and [6]) and is listed in Table 4. The vector $\underline{x}_{3}$ for $n=27$ is strongly suggested by the numerical minimization as Figure 3 shows, so that only a very limited search is required. We have also added vectors with the most pleasing fractions for $S-N / 2$, namely $\underline{x}_{\mathrm{int}}^{*}$ obtained from $\underline{x}_{\mathrm{int}}$ by changing $x_{9}$ to 31 , and $x_{3}^{*}$ by changing the first 10 in $\underline{x}_{3}$ to an 11 .

Table 4 lists the results of the numerical minimization and exhibits to high accuracy the relations conjectured in $\S 4$.

ACKNowledgment. The authors wish to thank P. H. Diananda for valuable information and suggestions.

\section{REFERENCES}

1. D. E. Daykin, Inequalities for functions of cyclic nature, J. London Math. Soc., (2), 3 (1971), 453-462.

2. P. H. Diananda, On a cyclic sum, Proc. Glasgow Math. Assoc., 6 (1963), 11-13. 
3. D. Ž. Djokovič, Sur une inégalité, Proc. Glasgow Math. Assoc., 6 (1963), 1-10.

4. C. V. Durell, Query, Math. Gaz., 40 (1956), 266.

5. M. Herschorn and J. E. L. Peck, Problem 4603, Amer. Math. Monthly, 67 (1960), $87-88$.

6. M. A. Malcolm, Note on a conjecture of L. J. Mordell, Math. Comp., 25 (1971), 375-377.

7. D. S. Mitrinović, Analytic Inequalities, (Springer-Verlag, New York 1970), 132-138.

8. P. Nowosad, Isoperimetric eigenvalue problems in algebra, Comm. Pure Appl. Math., 21 (1968), 401-465.

9. J. L. Searcy and B. A. Troesch, The cyclic inequality, Notices Amer. Math. Soc., 23 (1976), A-604.

10. D. G. Thomas, On the definiteness of certain quadratic forms arising in a conjecture of L. J. Mordell, Amer. Math. Monthly, 68 (1961), 472-473.

Received October 24, 1977 and in revised form October 24, 1978.

UNIVERSity of SoUthern CALIForNia

LOS ANGELES, CA 90007 


\section{PACIFIC JOURNAL OF MATHEMATICS}

EDITORS

DONALD BABBITT (Managing Editor)

University of California

Los Angeles, California 90024

HUGo RossI

University of Utah

Salt Lake City, UT 84112

C. C. MOORE

J. DUGUNDJI

Department of Mathematics

University of Southern California

Los Angeles, California 90007

R. FinN AND J. MiLgraM

Stanford University

Stanford, California 94305

University of California

Berkeley, CA 94720

\section{ASSOCIATE EDITORS}

E. F. BrCKENBACH

B. H. NeUmanN

F. WOLF

K. YoshidA

\section{SUPPORTING INSTITUTIONS}

UNIVERSITY OF BRITISH COLUMBIA

UNIVERSITY OF SOUTHERN CALIFORNIA

CALIFORNIA INSTITUTE OF TECHNOLOGY

STANFORD UNIVERSITY

UNIVERSITY OF CALIFORNIA

UNIVERSITY OF HAWAII

MONTANA STATE UNIVERSITY

UNIVERSITY OF TOKYO

UNIVERSITY OF NEVADA, RENO

UNIVERSITY OF UTAH

NEW MEXICO STATE UNIVERSITY

WASHINGTON STATE UNIVERSITY

OREGON STATE UNIVERSITY

UNIVERSITY OF WASHINGTON

UNIVERSITY OF OREGON 


\section{Pacific Journal of Mathematics}

\section{Vol. 81, No. $1 \quad$ November, 1979}

Thomas E. Armstrong, Simplicial subdivision of infinite-dimensional compact cubes ..................................... 1

Herbert Stanley Bear, Jr., Approximate identities and pointwise convergence ................................

Richard David Bourgin, Partial orderings for integral representations on convex sets with the Radon-Nikodým property..................

Alan Day, Herbert S. Gaskill and Werner Poguntke, Distributive lattices

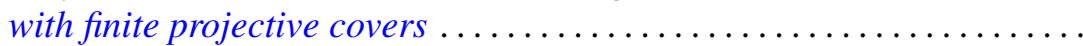

Heneri Amos Murima Dzinotyiweyi and Gerard L. G. Sleijpen, A note on measures on foundation semigroups with weakly compact orbits ......

Ronald James Evans, Resolution of sign ambiguities in Jacobi and Jacobsthal sums ...................................

John Albert Fridy, Tauberian theorems via block dominated matrices ......

Matthew Gould and Helen H. James, Automorphism groups retracting onto

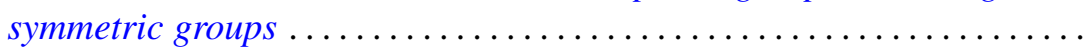

Kurt Kreith, Nonlinear differential equations with monotone solutions . . . . 101

Brian William McEnnis, Shifts on indefinite inner product spaces........ 113

Joseph B. Miles, On entire functions of infinite order with radially

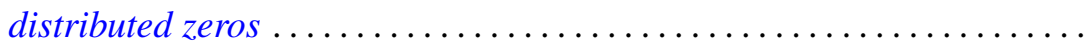

Janet E. Mills, The idempotents of a class of 0-simple inverse semigroups ...............................

Edward Jean Moulis, Jr., Generalizations of the Robertson functions ...

Richard A. Moynihan and Berthold Schweizer, Betweenness relations in probabilistic metric spaces.......................

Stanley Ocken, Perturbing embeddings in codimension two ....

Masilamani Sambandham, On the average number of real zeros of a class of random algebraic curves.

Jerry Searcy and B. Andreas Troesch, A cyclic inequality and a related eigenvalue problem.

Roger R. Smith and Joseph Dinneen Ward, $M$-ideals in $B\left(l_{p}\right)$...

Michel Talagrand, Deux généralisations d'un théorème de I. Namioka ..

Jürgen Voigt, $O n Y$-closed subspaces of $X$, for Banach spaces $X \subset Y$;

existence of alternating elements in subspaces of $C(J)$

Sidney Martin Webster, On mapping an $n$-ball into an $(n+1)$-ball in complex spaces

David J. Winter, Triangulable subalgebras of Lie p-algebras ... 